\title{
Increasing Children's Physical Activity by Policy (CAP) in Preschools Within the Stockholm Region: Study Protocol for a Cluster Randomized Controlled Trial
}

Chu Chen ( $\square$ Chen.chu@ki.se)

Karolinska Institutet https://orcid.org/0000-0001-6350-3797

Viktor H Ahlqvist

Karolinska Institutet

Pontus Henriksson

Linköping University

Jairo H. Migueles

Linköping University

Filip Christiansen

Karolinska Institutet

Maria Rosaria Galanti

Karolinska Institutet

Daniel Berglind

Karolinska Institutet

\section{Research Article}

Keywords: Physical activity, policy, intervention, preschool, accelerometer-based measurement

Posted Date: February 18th, 2022

DOl: https://doi.org/10.21203/rs.3.rs-998487/v1

License: (c) (1) This work is licensed under a Creative Commons Attribution 4.0 International License.

Read Full License 


\section{Abstract}

\section{Background:}

Systematic reviews suggest that preschool environmental/organizational changes may be effective in increasing physical activity (PA) levels of preschool children, but evidence is scarce regarding feasible, effective and equitable interventions that can be scaled up. Specifically, it is essential to understand whether introducing a multicomponent organizational change in terms of policy in the preschool context may be beneficial for children's PA levels and concomitant health outcomes. To bridge this knowledge gap, our main aim is to examine the feasibility and effectiveness of a policy package in increasing PA levels in preschool children, using a large scale pragmatic cluster randomized controlled trial.

\section{Methods:}

Ths proposed studyis a cluster-randomized trial with two conditions (intervention and control with a 1:1 ratio) with preschools as clusters and the unit of randomization. We aim to recruit approximately 4,000 35 years old children from 90 preschools and retain more than 2,800 chidlren from 85 preschools to provide adequate statistical power for the analyses. The intervention to implement is a co-created, multicomponent policy package running for 6 months in preschools randomized to intervention. Change in accelerometer measured PA levels in children between intervention and control from pre- and post intervention will be the primary outcome of the study, while secondary outcomes include health outcomes such as musculoskeletal fitness, psychosocial functioning and sick leave among others. Implementation will be studied carefully using both quantitative (dose, fidelity) and qualitative (interview) methodologies. The change in primary and and secondary outcomes, from pre- to post-intervention, will be analyzed with linear mixed-effect models (to allow both fixed and random effects) nested on preschool-level.

\section{Discussion:}

This is a large scale co-creation project involving the City of Stockholm, childcare stakeholders, preschool staff and the research group with the potential to influence more than 30,000 preschool children within the Stockholm area. The study will add reliable evidence for the implementation of PA policies at the organizational level of preschools, and clarify its potential effect on objectively measured PA and health markers in children.

\section{Trial Registration:}

This trial has been prostectively registered at clinicaltrials.gov with reference number NCT04569578 on September 20, 2020.

\section{Background}

Low levels of physical activity (PA) is a growing global concern (1) and efforts to increase PA are imperative for children of preschool age (2). Total PA and moderate to vigorous PA (MVPA) are positively 
associated with the healthy development of young children ( $<5$ years) (3). For example, childhood PA is positively associated with musculoskeletal fitness, cardiometabolic health and psychosocial health (3). In contrast, excessive sedentary time (ST) in youth is associated with increases in markers of cardiometabolic disease risk (4). Consistent data show that low levels of musculoskeletal fitness in children are longitudinally associated with low bone mass density, adiposity, metabolic risk factors and premature mortality (5). In addition, the healthy PA habits developed during early ages may track to later stages of life $(6,7)$. Consenquently, the assessment of PA and musculoskeletal fitness in youth are relevant from a public health perspective.

Despite the known benefits of PA, children in general engage in low levels of PA and their PA tends to decrease with age (8). A recent Swedish accelerometer-based national survey showed that only $23 \%$ of adolescent girls and $43 \%$ of boys reached the recommended level of a minimum of 60 minutes of daily MVPA (9). Moreover, low accelerometer measured PA levels have been shown in preschoolers, as highlighted by a study of approximately 1000 Swedish preschool children (10).

Higher intensity PA (e.g. MVPA) is a major determinant of phsycal fitness in children (11). In addition to the observed low levels of PA among preschoolers, physical fitness among youths is declining globally (12), and in Sweden (13). Notably, the decline in fitness level is more pronounced in countries with higher income inequality (12) where PA levels are lower in socioeconomically disadvantaged communities (9). Therefore, it is vital to develop strategies to promote PA in individuals with various socioeconomic backgounds, equitably as early as in preschool age.

The preschool setting presents unique opportunities to promote PA and health since the children are very young and habits can be more easily adquired. This makes this setting even more promising than the school setting. For example, preschool PA routines and practices can influence a major proportion of waking hours in children, since children in Sweden generally spend a large part of their waking hours at preschool. In addition, approximately $95 \%$ of Swedish 3-5 years-old children attend preschools regardless of their parent's socioeconomic status (SES) (14). Therefore, preschool interventions may offer each child an equitable opportunity in forming early healthy PA behaviors.

Moreover, preschools provide appropriate conditions to explore associations between PA, time spent outdoors and sick leave, which are important parameters from a public health as well as an economic perspective. For example, time spent outdoors at the preschool may contribute to health benefits through increased PA in children (15). In addition, increasing time spent outdoors may reduce person-to-person transmission of infections, since children brought together in close environments, such as a preschool indoor environment, are mutually exposed to the transmission of pathogens (16). It is possible that PA acts a mediator of the link between outdoor activities and sick leave (17); reduced infection rates could be achieved by reducing transmission of disease as well as reducing host susceptibility through the beneficial health effects of PA. However, the association between outdoor stay and sick leave is currently unclear, as outdoor time has been shown to both reduce (18) and increase (19) sick leave. In addition, research has shown equivocal effects from PA on sick leave (20). Nonetheless, it is conceivable that 
outdoors time impacts on infection rates by both decreasing the opportunity of transmission and by reinforcing immunity defense via PA. However, it is possible that outdoor activities also impact other morbidity (e.g. accidents and traumas); hence, the net effect may be concealed.

Previous meta-analyses and reviews of PA interventions in preschoolers indicate that interventions are likely to be effective when inducing environmental/organizational change in the preschool setting, targeting multicomponent factors such as integrated structured PA time, ensuring unstructured outdoor time and instructions to preschool teachers on how to lead structured activity $(21,22)$. Although policies may be potentialy modifiable organizational factors in preschools, introducing preschool policy can be challenging since preschool policies often suffer from unclearness, poor implementation, lack of tools for follow-up and measures of compliance $(23,24)$. Furthermore, few previous studies have explored the association between preschool PA policies and children's PA levels (25-27). An observational study from Sweden, have demonstrated higher PA levels in children enlisted in preschools with a PA policy (27). This result is in line with findings from other countries $(25,26)$. However, only a quarter of all participating preschools in the Swedish study had a PA policy (27). In general, the few existing randomized controlled trials within the field suffer from small sample sizes and limited intervention duration $(21,22)$. To the best of our knowledge, there is only one ongoing pilot randomized trial testing preschool PA policy on a small scale with a short duration of follow-up (28). Notably, although preschool teachers are key components in the implementation of policy, the policy's effect on preschool teachers' PA and its possible implications on parameters such as sick leave, has not been studied previously. Such knowledge could add to the understanding of preschool PA policies and how they may affect change in PA. In addtion, the equity perspective of PA interventions has been largely neglected previously (29).

\section{Objectives}

The primary aim of the increasing Children's physical Activity by Policy (CAP) study is to evaluate the feasibility and effectiveness of implementing PA policies in preschools on preschool children's PA levels. The secondary aims of the study include exploring the effectiveness of PA policy on preschool children's i) sedentary time; ii) screen time, iii) musculoskeletal fitness; iv) sleep; v) adiposity; vi) psychosocial functioning; vii) sick leave; viii) children's PA opportunity outside preschool time such as active transport and organized sports participation as well as on preschool teachers': i) physical activity levels, ii) sedentary time, iii) sleep iv) adiposity and v) sick leave. We will also investigate the implementation of the policy and how this policy can be normalized into preschool teachers' regular practice by exporing i) dose and fidelity of the implementation, ii) facilitators and barriers in implementing the preschool policy and iii) sustainability of the policy.

\section{Hypothesis}

The hypothesis is that implementing PA policies in preschools will increase PA in young children and preschool teachers equitably across districts with diverse SES. We further hypothesize that implementing PA policies will lead to improvements in children's musculoskeletal fitness, psychosocial functioning, sleep and reduce sedentary time, screen time and sick leave in both children and preschool teachers. The 
policy may also influence preschool children's PA opportunities outside preschool time such as increases in active transport. However, adiposity is hypothesized to not be affected as meta-analysis data showed limited evidence for an association between PA and adiposity among preschool children (30).

\section{Methods/design}

\section{Study design and setting}

The current study is a cluster randomized trial with two conditions (intervention and control) with preschools as clusters and the unit of randomization. A cluster randomization design was chosen because the intervention targets policy on the organizational level in preschools and preschools are natural clusters. Units of observations are preschool children and preschool teachers. The report of this trial protocol is guided by SPIRIT reporting guidelines (31) and we provide the detailed SPIRIT checklist (see Additional file 1). We will report the findings of the trial according to guidelines outlined in the CONSORT extension for cluster randomized trials (32). The current study is being conducted in the Stockholm region of Sweden. To date in the Stockholm region, there are 3,0969 children (1-5 years old) enrolled in a total of 539 public preschools (personal communication with Stockholm region representative, 2020-06-15). The Stockholm region can be divided geographically and administratively into 13 districts which vary in size, number of preschools, children and resources.

\section{Ethical approvals and informed consent}

\section{Ethical approval}

has been obtained from the Swedish Ethical Review Authority (Dnr. 2020-03002) and this study has also been pre-registered at a database for clinical studies (https://clinicaltrials.gov) with reference number: NCT04569578. All items from the World health Organization Trial Registration Data Set can be found clinicaltrials.gov. The registration of the study was completed before the recruitment of participants. An information document, describing the study purpose and procedures, will be provided to every participant and further information is provided on the project website (https://ces.sll.se/var-

Thverksamhet/aktiviteter-och-projekt/rorelseprojektet/). Written informed consent will be obtained from parents of the participating children and participating preschool teachers before enrollment in the study. (See Additional file 2 for the consent form in English) Please refer to Additional file 3 for the details about how the informed consent will be collected.

\section{Sample size}

The required sample size to obtain a statistical power of 0.90 was projected with a change in MVPA as the main outcome variable and by using the means and standard deviations of the aforementioned observational preschool study assessing PA (27). A 5 minute change in MVPA has been suggested in previous meta-analysis to be a resoable target a of clinical importance (33). Assuming an effect size of 5 min MVPA, an an intra-preschool correlation of 0.2 , a standard deviation of 25 , coefficient of variation of 0.3 (average preschool size of 35 children 3-5 years), approximaley 2800 children (from 85 preschools) should be retained. 


\section{Study population and recruitment}

Based on other similar studies and the previously mentioned preschool study conducted by our research group (27), approximately $30 \%$ total non-participation is expected. Specifically, we expect an estimated accelerometer non-compliance rate of $10 \%$ (i.e. participants with insufficient wear-time) and a drop-out rate of $20 \%$ throughout the study period. Consequently, we aim to recruit approximately 4000 children aged 3-5 years from 100 public preschools within all 13 districts in the Stockholm region.

\section{Inclusion/exclusion criteria}

Figure 1 illustrates the recruitment of preschools and subsequent recruitment of preschool children and teachers based on the inclusion/exclusion criteria. Due to the logistical burden and time limit of the study, only preschools with more than 60 enlisted children are eligible for participation. In order to ensure a representative sample in each district, approximately $30 \%$ of all public schools with more than 60 enlisted children (all age groups) in each district will be randomly asked to participate in the study. The random selection of preschools for study invitation will be fulfilled by contacting preschool principals following a random order of invitation generated by Stata (Additional file 4. programming codes, random_invatation_preschool) until the number of preschools agreed to participate reached the approximately $30 \%$ of all public preschools in that district. The recruitment of preschool children and teachers will be initiated provided that preschool principals agree to participate. All 3-5 years old children enlisted at the participating preschools will be invited to participate and the participation will be confirmed by the informed consent of parents. In addition, full-time teachers at the participating preschools will also invited to participate and provide informed consent. Preschools with less than 10 children with parental informed consent and children who are not able to able to take part in PA measures (e.g. mobility disability) will be excluded from participation. The limit of at least 10 children with parental informed consent per preschool was chosen due to logistical reasons to accommodate the accelerometer measurement burden in the study.

A total of 156 preschools were asked to participate in the study and 127 preschools (with median of 11 preschools per district) agreed to participate. Apart from 3 preschools that collected less than 3 parental informed consents, 124 preschools participated in the baseline data collection. No preschools were lost to follow up.

\section{Randomization and blinding}

Preschools will be stratified by the 13 Region Stockholm districts because geographical, organizational and resource factors in each district are likely to differ and influence the implementation and effectiveness of the policy. After baseline assessment, preschools will be randomly assigned to the intervention or control condition by 1:1 ratio within each of the 13 districts to ensure equal representation of intervention and control preschools. Block randomization with districts as blocks is conducted with randomizr package in $\mathrm{R}$ (Version3.6.1) (Additional file 5. programming codes: randomization_preschool). 
A single-blinded approach will be adopted where outcome assessors will be blinded to the treatment allocation.

\section{Intervention description}

The intervention components were developed based on the aforementioned observational preschool study (27) and systematic review data on the effectiveness of PA interventions for preschoolers, i.e. (i) organizational changes at the policy level, (ii) unstructured outdoor play, and (iii) instructions to preschool personnel on how to encourage child PA behaviors (21). The intervention framework, a study website and delivery of information, has been established on the directional management level within all 13 districts within the Stockholm region and will be delivered directly to each intervention preschool after baseline measures and the randomization process. To increase parental engagement in the intervention group, participating parents will be provided with an information package (multi-lingual multi-media content) describing the intervention components, their importance, and practical information on how to engage in active transportation to/from preschool.

The intervention framework (on preschool level) includes:

i. Formalized PA policies:

a. A minimum of 3 hours of total daily outdoor time, to be scheduled both in the morning and in the afternoon.

b. At least 10 minutes of teacher-led active play per day. An activity bank will be provided for inspirations of possible teacher-led activities.

c. A minimum of 1 weekly outdoor excursion which is commonly realized as a visit to local parks, playgrounds or other recreational facilities. A map of recommendations of potential outdoor excursion areas will be provided.

d. Meet the children outdoors when they arrive at preschool and/or picked up by their parents.

e. Only use screen-based devices for educational teaching purposes.

f. Communicating to, and encouraging, the parents to actively transport their children to and from the preschool.

ii. A study website will provide information on the study process and measurements. The activity bank for inspiration of teacher-led active play and a map of recommendations for outdoor exertion, mentioned in PA policy $b$ and $c$ above, will also be accessible through the website. This study website may also serve as a platform for interprofessional education where preschool teachers can share and communicate about engagement in activities and their experience.

iii. Weekly follow-up and feedback by web-based questionnaires to determine fidelity and dose of intervention.

\section{Intervention development and theory base}


The interest for the intervention began when the previously mentioned observational study, found a positive association between having a formalized PA policy in preschools and children's PA levels (27), which attracted attention from politicians and stakeholders in the City of Stockholm. Realizing the potential of policy to increase PA among preschool children, the stakeholders took a formal political decision that all public preschools in the Stockholm region should implement a formalized PA policy. This decision is documented in the public preschools' financial steering plan and implies that all public preschools in the Stockholm region should exert effort and financial resources in the implementation of a PA policy. In addition, a policy package should be developed and evaluated to provide a "model" for preschools to understand what PA policies to implement and effective strategies for implementation. The intervention policy package has been developed in co-creation among the research group, municipal childcare stakeholders, district officers from the 13 Stockholm region districts and a reference group consisting of preschool principals and teachers. The policy package has been discussed and acknowledged among all co-creators to increase the engagement, buy-in, feasibility, relevance and sustainability of the intervention policy package as recommended by a previous meta-analysis on the effectiveness in co-creation of research (34). The Behaviour Change wheel (35) was chosen as a theory base to understand and reflect on essential conditions (capacity, opportunity and motivation) for behaviour change in relation to the intervention's functions and policy category. (The detailed logical model of theory of change for the policy package intervention based on behavioral change wheel can be found in Additional file 6 theory of change).

\section{Implementation of the intervention}

This intervention is expected to scale up upon proof of effectiveness and feasibility. Therefore, no special implementation facilitation will be provided - to ensure external validity. Specifically, to ensure complete scalability we will utilize a pragmatic approach where minimal additional resources are provided (no support beyond that of the intervention package), thereby evaluating whether the routine resources disposable to Swedish preschools are sufficient for the implementation of the intervention. The implementation of the policy package will be examined in detail following the process evaluation framework by Moore et al (36). To which extent the policy package is implemented (dose and fidelity) is followed up weekly by web-based questionnaires. More details of process evaluation are illustrated in the process outcomes section.

\section{Control condition}

Preschools randomized to the control condition are not provided with the intervention package and are encouraged to keep their usual practice and routines during the intervention period. However, in this pragmatic trial, control preschools are also under the influence of financial steering document and they are free to implement any PA policy. In addition, communication between teachers from intervention and control groups and the study website may contribute to contamination. Therefore, PA policies in control preschools will be monitored at baseline, mid-point ( 3 months) and endpoint ( 6 months) to capture possible contamination effects and understand the possible change in their PA policy throughout the intervention. 


\section{Outcomes and measures}

In the present study, several instruments are used to assess outcomes in children and teachers. An additional file provides an illustration of all the instruments used, corresponding to the measured outcome variable, time point of measurement and group relevance (i.e measurement in both intervention and control groups or intervention group only) in detail (See Additional file 7). Questionnaires will be distributed via email to each respondent through Webropool 3.0. All questionnaires used can be found in Supplementary material 3 questionnaires. Questionnaires answered by preschool personnel are distributed only in Swedish. The questionnaires distributed to parents are available in Swedish, English, Arabic, Turkish, Somali, Tigrinya and Swahili to cater to the need of parents with language barriers as an effort to retain a study sample containing individuals from different countries of origins. The questinnaires included as additional files (see Additional file 8-15) are only available in english and questionnairs in other languages can be made available upon reasonable request. How the data will be managed after collection and plan for result dissemination can be found in Additional file 3 .

\section{Physical activity levels (primary outcome) and sedentary time (secondary outcome) in children}

PA levels in children such as total PA, MVPA, light PA (LPA), ST and steps will be measured objectively using triaxial GT3X+ accelerometers, which have been tested extensively for reliability and validity in both pediatric and adult populations (37). Children will be instructed to wear the accelerometer, on the nondominant wrist, for 24 hours during 7 consecutive days except during water activities (e.g. when showering and swimming) at both baseline and 6 months follow-up. The non-dominant hand will be predetermined by parents (information collected together with informed consent). Set-up and analysis of accelerometer data will follow validated age-specific criteria and recommendations (37). The accelerometer data sampling frequency is $30 \mathrm{~Hz}$ and data will be analyzed at 1-s epochs which suits the sporadic movement pattern of preschool children (37). Raw accelerometer data will be downloaded through Actilife and analyzed using the GGIR package (38). The classification of PA intensities and ST will follow the cut-offs developed for children with wrist-worn Actigraph GT3X+ by Hildebrand et al (39, 40). Steps will be determined using the Verisense step algorithm, which is integrated in GGIR to determine steps for wrist-worn accelerometry (41). Accelerometer data consisting of $\geq 10$ hours/day wear time with $>3$ days of measurement days will be considered valid (42). However, participants with less valid days will be included in sensitivity analysis.

Results will be reported in categories of PA during the preschool time, PA outside preschool time and PA during the entire day. This is to explore how the preschool policy affects PA during the preschool time frame and to assess potential extended effect beyond the preschool time frame and compensatory behavior (43). Change in MVPA will be emphasized since PA in higher intensity is suggested to have greater benefits for health outcomes (3).

Musculoskeletal fitness in children (secondary outcome) 
Musculoskeletal fitness, in terms of handgrip strength, will be measured by an analogue dynamometer (TKK 5825, Grip-A, Takei, Tokyo, Japan) validated in preschool-aged children (44). Measurement of handgrip strength will be conducted at baseline and at 6 months follow-up by trained field workers to the nearest $0.1 \mathrm{~kg}$ on both dominant and non-dominant hands.

\section{Psychosocial functioning of children (secondary outcome)}

The psychosocial functioning of children will be assessed by a parental report of the Strength and Difficulty Questionnaire (SDQ) which has been used extensively worldwide and validated also in Swedish settings (45). Parents will be asked to fill in the SDQ and sleep questionnaire at both baseline and 6 months follow-up. The SDQ questionnaire uses a 3-point Likert scale to measure the children's psychosocial strength and problems summarized into an emotional symptoms scale, conduct problems

scale, hyperactivity scale, peer problem scale and prosocial scale. All scales except for the prosocial scale are then summarized into a total difficulties score. The result for psychosocial functioning will be reported as the total difficulties score as well as separate scores on each of the scales mentioned above.

\section{Sleep of children (secondary outcome)}

Sleep will be measured using a combination of $24 \mathrm{~h}$ accelerometry, a sleep time diary during periods of accelerometer measurements and a parental questionnaire to reflect sleep quality during the past 6 months. All measurements for sleep will be conducted at baseline and 6 months follow-up. Sleep onset, sleep duration and waking up will be determined by algorithms integrated in GGIR (46). Appropriate algorithms for detection of sleep in children will be used. The sleep time diary included in the parental measurement week questionnaire consists of two questions regarding children's wake up time and bedtime. In addition, parents will answer questions in SDQ and sleep questionnaire about children's sleep duration and quality during the past 6 months and these questions are adapted from the validated Ages and Stages Questionnaire (47). To minimize the burden of answering different questionnaires, the sleep quality questions were added to the SDQ questionnaire; therefore, the questionnaire is named SDQ and sleep questionnaire.

\section{Screen time in children (secondary outcome)}

Children's screen time, in minutes of screen time per day, will be reported by parents at baseline and endpoint during periods of accelerometer measurements in the parental measurement week questionnaire.

\section{Sick leave of children (secondary outcome)}

Sick leave frequency and duration from 12 months prior to the intervention baseline and during the 6months intervention period (18 months in total) will be collected from the the City of Stockholm central preschool absence database. A database to which all parents of preschool children in Stockholm report all sick leave.

\section{Adiposity of children (secondary outcome)}


Weight, height and waist circumference will be measured, by trained field workers, at baseline and 6 months follow-up. All measurements will be conducted twice and each measurement result will be recorded to the nearest 0.1 of the respective unit. Weight will be measured by validated scales (calibrated scale: VB2-200-EC, Vetek AB, Väddö, Sweden) and height will be measured by stadiometers (portable stadiometer: Seca 213, Seca, Chino, CA, USA). Weight and height will then be used to calculate body mass index $\left(\mathrm{kg} / \mathrm{m}^{2}\right)$ and children will be classified as normal weight, overweight or obese based on body mass index criteria developed by Cole et al (48). Waist circumference will be measured at the level of the navel directly on the skin (49). Due to the COVID-19 pandemic field researchers are required to conduct the measurements outdoors; thus, the waist circumference measurement may be conducted over thin clothing in cold weather.

\section{Demographic information, children and parents (descriptive)}

Information on the age and sex of parents of the participating children will be derived from the personal registry number provided upon the consent of participation. Parents will also, at baseline, be asked to complete a descriptive information questionnaire including questions regarding their education level, occupation, country of birth, height and weight.

\section{Children's PA opportunities outside preschool time (process outcome)}

Children's PA opportunities including participation in organized sports and active transport to/from preschool will be assessed via parental measurement week questionnaire (during the weeks of accelerometer measures at baseline and 6 months follow-up). Parents will be asked about the time, duration and type of organized sports, and mode of transportation to/from preschool. In addition, parents will also answer an active transport questionnaire at baseline and 6 months follow-up regarding children's general frequency of active transport, mode of transport, parental perception of the distance and condition of transportation route during the past 6 months. Moreover, several parental practices (outdoor time during the weekend, parental perception of PA, parental PA levels, and active play with parents) which could potentially influence children's daily PA levels will also be assessed by questions included in the parental questionnaire.

\section{Parental evaluation of the project and interview (process outcome)}

A parental evaluation questionnaire will be distributed to parents of all participating children at 6 months follow-up. This evaluation questionnaire includes the parental rating (5 score scale) and open-ended questions on information quality, communication and perception study influence and experiences of study participation. Parents have the opportunity to report any unintended adverse effects of the intervention in this instrument. These questions will be used in qualitative analysis of potential facilitators and barriers of the study. At the end of this questionnaire, parents will be asked whether they want to participate in an interview to further explore the experience, influence and potential of the study. 


\section{Physical activity levels, sedentary time and sleep in preschool teachers (secondary outcome)}

Similar to children's PA levels, ST and sleep, these variables in teachers will also be measured objectively using triaxial GT3X+ accelerometers (37). Preschool teachers will wear the accelerometer, on the nondominant wrist, for 24 hours during 7 consecutive days at baseline and 6 months follow-up. Minutes in total PA, MVPA, LPA, ST and total steps during the preschool time, outside preschool time and during the whole day will be reported for teachers. Sleep in preschool teachers will also be analyzed using $24 \mathrm{~h}$ accelerometry. Set-up and analysis of accelerometer data will be performed following validated agespecific criteria and recommendations (37).

\section{Musculoskeletal fitness of preschool teachers (secondary outcome)}

The method to assess musculoskeletal fitness in children will be used also for preschool teachers. Musculoskeletal fitness in terms of handgrip strength will be measured by an analogue dynamometer (TKK 5825, Grip-A, Takei, Tokyo, Japan). Measurement of handgrip strength will be conducted at baseline and at 6 months follow-up by trained field workers and recorded to the nearest $0.1 \mathrm{~kg}$ on both dominant and non-dominant hands.

\section{Sick leave of preschool teachers (secondary outcome)}

Sick leave frequency and duration from 12 months prior to the intervention baseline and during the 6months intervention period (18 months in total) will be collected from the City of Stockholm central preschool absence database.

\section{Adiposity of preschool teachers (secondary outcome)}

Weight and height will be measured by validated scales (calibrated scale: VB2-200-EC, Vetek AB, Väddö, Sweden) and stadiometers (portable stadiometer: Seca 213, Seca, Chino, CA, USA) and waist circumference will be measured at the level of the navel directly on the skin (49). Due to the COVID-19 pandemic field researchers are required to conduct the measurements outdoors; thus, the waist circumference measurement may be conducted over thin clothing in cold weather.

\section{Demography of preschool teachers (descriptive)}

The age and sex of the preschool teachers will be derived from their personal registry number provided upon consent to participate in the study.

\section{Preschool policy and environmental characteristics (process outcome)}

Preschool environmental/organizational characteristics such as available play equipment, playground characteristics, current policies and working practices will be assessed by the validated Environment and 
Policy Evaluation and Observation as a Self-Report Instrument (EPAO-SR) (50). The preschool playground characteristics and size will be double-checked and measured objectively via spatial mapping using publicly available satellite imagery (e.g. Google maps). Preschool level routine screen time will be reported by preschool teachers in intervention preschools weekly through the intervention weekly followup questionnaire and via the validated EPAO-SR (50). The use of EPAO-SR is important to identify what policies/practices that are in place prior to the intervention, and to assess if any other preschool environmental/organizational changes take place during the intervention period. It is also a crucial tool to measure the extent of any contamination by the intervention to the control preschools.

\section{Implementation of the policy package (process outcome)}

Fidelity and dose of the implemented policy package will be documented through a weekly follow-up questionnaire, answered by preschool staff, throughout the intervention period. The extent to which the intervention can be normalized into preschool staff's regular practice will be assessed through the validated (51) No-MAD questionnaire (an instrument for assessing implementation work based on normalization process theory) (52) at 6 months follow-up.

\section{Preschool teachers' evaluation of the project (process outcome)}

Communication, acceptance, facilitators and barriers for implementation will be assessed through teachers' evaluation questionnaires provided by all participating preschool teachers. At the end of the teachers' evaluation questionnaire, preschool teachers will be asked whether they are willing to participate in focus groups and interviews. Focus groups and interviews with preschool personnel will also be undertaken post-intervention to collect more in-depth perspectives regarding the adequacy of the intervention, the feasibility of implementation and suggestions for improvements. These qualitative discussions will generate rich data regarding the logistical challenges and the strengths and weaknesses of the intervention that could be missed in questionnaires. An experienced moderator, using a semistructured interview guide, will facilitate the discussions. Sample questions will include:

- What challenges did you experience when implementing the intervention?

- What solutions did you undertake to deal with these challenges?

- What characteristics of the intervention do you feel were most appropriate for increasing PA participation among preschoolers?

Focus groups and interviews will be audio-recorded and transcribed verbatim.

\section{Statistical analysis}

The baseline demographic characteristics of the participants will be summarized descriptively. Drop out participants' characteristics will be explored by comparison to the non-drop-outs. Fidelity and dose of implementation will be summarized as a score (fidelity $x$ number of weeks of compliance). The change in PA levels and secondary outcomes, from pre- to post-intervention, will be analyzed with linear mixedeffect models (to allow both fixed and random effects) nested on preschool level. The linear mixed- 
effects model can test several aspects of the study effects, e.g. effects over time inside a group, and groups against each other at a given time (e.g. intervention vs. control at 6-months follow-up). Subgroup analyses will be conducted to analyze the effect of the intervention in relation to socioeconomic aspects. Furthermore, we aim to explore if the COVID-19 pandemic has modified any implementation aspects of the intervention (via questionnaires on how various routines including outdoor time, hygiene aspects at preschools, homestay and transportation mode to/from preschool have changed following the COVID-19 pandemic).

\section{Timeplan of the project}

Figure 2 illustrates the schedule of enrollment, interventions, and assessments according to SPIRIT guidence.

\section{Discussion}

The CAP study aims to evaluate the feasibility and effectiveness of implementing a formalized policy package in Stockholm public preschools to increase levels of PA in preschool children. This is an unprecedented co-creation project among the City of Stockholm, childcare stakeholders, preschool staff, and the research group with the potential to finally impact levels of PA in more than 30,000 preschoolers, in more than 500 preschools in the Stockholm area. The project has received media and political attention which has led to a formal political decision that all 539 public preschools within the Stockholm region should implement a formalized PA policy (included in the public preschool financial steering document). To ensure evidence-based policymaking, it is imperative to evaluate the feasibility and effectiveness of this PA policy implementation. To the best of our knowledge, there is no other large-scale pragmatic cluster-randomized intervention study on preschool organizational level, evaluated with robust instruments in Sweden or elsewhere. This project will contribute to the implementation of PA policies at the organizational level of preschools, by providing reliabel evidence of its effect on objectively measured PA and health markers in children, through an equity perspective.

The main strengths of this study include the cluster randomized controlled study design and a large sample size, particularly for preschool-aged children who imperatively need scalable effective interventions to improve their PA. In addition, the co-creation of the policy package among the research group, municipal childcare stakeholders, district officer's from the 13 Stockholm region districts and a reference group consisting of preschool directors and preschool teachers will likely increase the buy-in, feasibility, relevance and sustainability of the intervention policy (34). Moreover, this study is a pragmatic trial without implementation facilitation and with the control group observed in "usual conditions", i.e. left "free" to implement any policy. This kind of pragmatic approach evaluates the policy package in a real life setting and provides insight into the effectiveness of a co-created policy package in increasing PA levels in preschool children. This will provide valuable information for decision-makers regarding feasibility, effectiveness and the conditions for scaling up. Another major strength of the present study is the objectively measured PA, which increases reliability and validity of data compared to subjective 
assessment (37). Finally, in order to generalize the results to a broader population and address the lack of equity perspective in previous studies, the present study covers multiple districts with variations in socioeconomic status, and the study information and questionnaires have been translated into most of the major languages spoken in Sweden.

There are several potential limitations of the planned study. First, the PA policy initiative, included in the preschool financial steering document, was launched before the baseline measurement. Hence, the baseline measurement may be affected by this initiative. Second, although only intervention preschools are provided with the intervention material, contamination may occur since the communication between staff from different preschools cannot be excluded. However, the policy in place and preschool practices will be measured closely in both intervention and control preschools at baseline, mid-point and end-point of the study to monitor and understand the effect of an active control group and possible contamination. The effects of the intervention may be underestimated due to these limitations, but a detailed understanding of these limitations give insights regarding the effectiveness of a policy intervention under real-life conditions. Third, the ongoing COVID-19 pandemic and accompanying societal strategies (e.g. social distancing recommendations) to limit the spread of COVID-19 has brought restrictions on daily living and changes in preschool routines. More specifically, preschools in Sweden are recommended to increase the outdoor time during preschool hours. This change is likely to influence the study results through increased PA since extended outdoor time is an important component of the intervention. Nevertheless, it is hard to perceive and predict the future development of COVID-19 and its potential influence during the course of the study. To capture potential COVID-19 pandemic effects on intervention components (e.g. time spent outdoors), web-based questionnaires will be delivered to both participating control and intervention preschools teachers and parents. The questionnaires will contain questions on how various routines including outdoor time, hygiene aspects at preschools, homestay and transportation mode to/from preschool have changed following the COVID-19 pandemic. It is also possible that the COVID-19 pandemic has increased awareness of the importance of health, PA and outdoor time in the general population. Thus, the preschool personnel may be more motivated to implement and sustain the policy. These potential effects will be captured by program evaluation questionnaires and interviews.

This study will provide robust evidence of the effect of organizational changes in preschools on children's PA and indicators of health. Notably, such robust evidence is urgently warranted as Swedish preschool children's PA levels are currently low, which may impact their long-term health. Finally, as the intervention has been developed in co-creation with the City of Stockholm it has the possibility to, if proven effective, be implemented rapidly within preschools.

\section{Trial Status}

This is the first version of protocol submitted on 2021-10-18. Recruitment of the participants started 2020-09-23 and the last visit to preschool was on 2021-07-02. However, not all data has currently been collected due to delay in returning of acclerometers from participants and interviews with participants are 
under planning. The trial protocol was finalized before any data collected from measurement was processed and important changes to the protocol, if any, will be communicated to the journal and trial registry.

\section{List Of Abbreviations}

physical activity (PA)

moderate to vigorous physical activity (MVPA)

sedentary time (ST)

socioeconomic status (SES)

increasing Children's physical Activity by Policy (CAP) study

Strength and Difficulty Questionnaire (SDQ)

Environment and Policy Evaluation and Observation as a Self-Report Instrument (EPAO-SR)

\section{Declarations}

\section{Ethics approval and consent to participate}

Ethical approval has been obtained from the Swedish Ethical Review Authority (Dnr. 2020-03002) and written informed consent will be obtained from parents of the participating children and participating preschool teachers before enrollment in the study.

\section{Consent for publication}

Not applicable

\section{Availability of data and materials}

There is no data included in this study protocol. The data collected in this study will be stored in the central database in Centre for Epidemiology and Community Medicine (CES). In the future publication regarding trial results, we aim for open access for the public access of knowledge and all statistical codes will be made available. Dataset cannot be made publicly available due to restriction in Ethical approval unless additional approval is granted for reasonal request by the Ethical review committee.

\section{Competing interests}

All authors declare no competing interests.

\section{Funding}


This study was funded by The Health and Medical Care Administration (Hälso- och sjukvårdsförvaltningen, Region Stockholm) and The Swedish Research Council for Sport Science (grant given to PI Daniel Berglind).

The Health and Medical Care Administration can be reached via email registrator.hsf@regionstockholm.se and The Swedish Research Council for Spoer Science can be contadcted via email cif@gih.se or postal address Centrum för idrottsforskning, Box 5626, S-114 86 Stockholm. All funding parties have no role in study design, collection, management, interpretation or dissenmination of data.

\section{Authors' contributions}

Chu Chen: draft protocol manuscript, data collection/management. Viktor H. Ahlqvist: Edite protocol manuscript, contribute to sample size calculation. Pontus Henriksson and Jairo H. Migueles: Edit protocol manuscript, especially accelerometer data collection setup and process. Filip Christiansen: data collection, protocol manuscript editing. Maria Rosaria Galanti: study design, protocol manuscript editing. DB: study design, draft protocol. All authors read and approved the final manuscript.

\section{Acknowledgements}

We would like to thank participating children, their parents and preschool teachers/staff for their participation in the study.

\section{References}

1. Guthold R, Stevens GA, Riley LM, Bull FC. Worldwide trends in insufficient physical activity from 2001 to 2016: a pooled analysis of 358 population-based surveys with 1.9 million participants. The Lancet Global Health. 2018;6(10):e1077-e86.

2. Tremblay MS, Gray CE, Akinroye K, Harrington DM, Katzmarzyk PT, Lambert EV, et al. Physical activity of children: a global matrix of grades comparing 15 countries. J Phys Act Health. 2014;11(Suppl 1):113-25.

3. Carson V, Lee EY, Hewitt L, Jennings C, Hunter S, Kuzik N, et al. Systematic review of the relationships between physical activity and health indicators in the early years (0-4 years). BMC Public Health. 2017;17(Suppl 5):854.

4. Saunders TJ, Chaput JP, Tremblay MS. Sedentary behaviour as an emerging risk factor for cardiometabolic diseases in children and youth. Can J Diabetes. 2014;38(1):53-61.

5. Smith JJ, Eather N, Morgan PJ, Plotnikoff RC, Faigenbaum AD, Lubans DR. The health benefits of muscular fitness for children and adolescents: a systematic review and meta-analysis. Sports medicine. 2014;44(9):1209-23.

6. Jones RA, Hinkley T, Okely AD, Salmon J. Tracking Physical Activity and Sedentary Behavior in Childhood: A Systematic Review. Am J Prev Med. 2013;44(6):651-8. 
7. Huotari P, Nupponen H, Mikkelsson L, Laakso L, Kujala U. Adolescent physical fitness and activity as predictors of adulthood activity. Journal of sports sciences. 2011;29(11):1135-41.

8. Steene-Johannessen J, Hansen BH, Dalene KE, Kolle E, Northstone K, Moller NC, et al. Variations in accelerometry measured physical activity and sedentary time across Europe - harmonized analyses of 47,497 children and adolescents. Int J Behav Nutr Phys Act. 2020;17(1):38.

9. Nyberg G, Kjellenberg K, Fröberg A, Lindroos AK. A national survey showed low levels of physical activity in a representative sample of Swedish adolescents. Acta paediatrica.n/a(n/a).

10. Berglind D, Tynelius P. Objectively measured physical activity patterns, sedentary time and parentreported screen-time across the day in four-year-old Swedish children. BMC Public Health. 2017;18(1):69.

11. Ortega FB, Ruiz JR, Castillo MJ, Sjostrom M. Physical fitness in childhood and adolescence: a powerful marker of health. Int J Obes (Lond). 2008;32(1):1-11.

12. Tomkinson G, Lang J, Tremblay M. Temporal trends in the cardiorespiratory fitness of children and adolescents representing 19 high-income and upper middle-income countries between 1981 and 2014. Br J Sports Med. 2017;53:bjsports-2017.

13. Westerstahl M, Barnekow-Bergkvist M, Hedberg G, Jansson E. Secular trends in body dimensions and physical fitness among adolescents in Sweden from 1974 to 1995. Scand J Med Sci Sport. 2003;13(2):128-37.

14. Skolverket. Förskolbarn och personal https://www.skolverket.se/skolutveckling/statistik/2019 [.

15. Boldemann C, Blennow M, Dal H, Martensson F, Raustorp A, Yuen K, et al. Impact of preschool environment upon children's physical activity and sun exposure. Prev Med. 2006;42(4):301-8.

16. Forssell G, Hakånsson A, Månsson N-O. Risk factors for respiratory tract infections in children aged 2-5 years. Scand J Prim Health Care. 2001;19(2):122-5.

17. Chastin SFM, Abaraogu U, Bourgois JG, Dall PM, Darnborough J, Duncan E, et al. Effects of Regular Physical Activity on the Immune System, Vaccination and Risk of Community-Acquired Infectious Disease in the General Population: Systematic Review and Meta-Analysis. Sports Med. 2021;51(8):1673-86.

18. Bondestam M, Rasmussen F. Preschool children's absenteeism from Swedish municipal day-care centres because of illness in 1977 and 1990. Geographical variations and characteristics of the daycare centres. Scand J Soc Med. 1994;22(1):20-6.

19. Mygind O, Rønne T, Søe A-L, Wachmann CH, Ricks P. Comparative intervention study among Danish daycare children: the effect on illness of time spent outdoors. Scandinavian journal of public health. 2003;31(6):439-43.

20. Amlani NM, Munir F. Does physical activity have an impact on sickness absence? A review. Sports medicine. 2014;44(7):887-907.

21. Gordon ES, Tucker P, Burke SM, Carron AV. Effectiveness of physical activity interventions for preschoolers: a meta-analysis. Res Q Exerc Sport. 2013;84(3):287-94. 
22. Messing S, Rutten A, Abu-Omar K, Ungerer-Rohrich U, Goodwin L, Burlacu I, et al. How Can Physical Activity Be Promoted Among Children and Adolescents? A Systematic Review of Reviews Across Settings. Frontiers in public health. 2019;7:55.

23. Coe DP. Means of Optimizing Physical Activity in the Preschool Environment. Am J Lifestyle Med. 2020;14(1):16-23.

24. Kracht CL, Webster EK, Staiano AE. A natural experiment of state-level physical activity and screentime policy changes early childhood education (ECE) centers and child physical activity. BMC Public Health. 2020;20(1):387.

25. Bower JK, Hales DP, Tate DF, Rubin DA, Benjamin SE, Ward DS. The childcare environment and children's physical activity. Am J Prev Med. 2008;34(1):23-9.

26. Bell AC, Finch M, Wolfenden L, Fitzgerald M, Morgan PJ, Jones J, et al. Child physical activity levels and associations with modifiable characteristics in centre-based childcare. Aust N Z J Public Health. $2015 ; 39(3): 232-6$.

27. Chen C, Ahlqvist VH, Henriksson P, Magnusson C, Berglind D. Preschool environment and preschool teacher's physical activity and their association with children's activity levels at preschool. PLoS One. 2020;15(10):e0239838.

28. Tucker P, Driediger M, Vanderloo LM, Burke SM, Irwin JD, Johnson AM, et al. Exploring the Feasibility and Effectiveness of a Childcare PhysicaL ActivitY (PLAY) Policy: Rationale and Protocol for a Pilot, Cluster-Randomized Controlled Trial. International journal of environmental research and public health. 2019;16(22).

29. Lehne G, Brand T, Bolte G. Equity impact of interventions promoting physical activity - points to consider: Gesa Lehne. European Journal of Public Health. 2017;27(suppl_3).

30. Wiersma R, Haverkamp BF, van Beek JH, Riemersma AMJ, Boezen HM, Smidt N, et al. Unravelling the association between accelerometer-derived physical activity and adiposity among preschool children: A systematic review and meta-analyses. Obes Rev. 2020;21(2):e12936.

31. Chan A-W, Tetzlaff JM, Gøtzsche PC, Altman DG, Mann H, Berlin JA, et al. SPIRIT 2013 explanation and elaboration: guidance for protocols of clinical trials. BMJ: British Medical Journal. 2013;346:e7586.

32. Campbell MK, Piaggio G, Elbourne DR, Altman DG, Group C. Consort 2010 statement: extension to cluster randomised trials. BMJ. 2012;345:e5661.

33. Metcalf B, Henley W, Wilkin T. Effectiveness of intervention on physical activity of children: systematic review and meta-analysis of controlled trials with objectively measured outcomes (EarlyBird 54). BMJ. 2012;345:e5888.

34. Halvorsrud K, Kucharska J, Adlington K, Rüdell K, Brown Hajdukova E, Nazroo J, et al. Identifying evidence of effectiveness in the co-creation of research: a systematic review and meta-analysis of the international healthcare literature. Journal of public health. 2019.

35. Michie S, van Stralen MM, West R. The behaviour change wheel: a new method for characterising and designing behaviour change interventions. Implement Sci. 2011;6:42. 
36. Moore GF, Audrey S, Barker M, Bond L, Bonell C, Hardeman W, et al. Process evaluation of complex interventions: Medical Research Council guidance. BMJ. 2015;350:h1258.

37. Migueles JH, Cadenas-Sanchez C, Ekelund U, Delisle Nystrom C, Mora-Gonzalez J, Lof M, et al. Accelerometer Data Collection and Processing Criteria to Assess Physical Activity and Other Outcomes: A Systematic Review and Practical Considerations. Sports medicine. 2017.

38. Migueles JH, Rowlands AV, Huber F, Sabia S, van Hees VT. GGIR: A Research Community-Driven Open Source R Package for Generating Physical Activity and Sleep Outcomes From Multi-Day Raw Accelerometer Data. Journal for the Measurement of Physical Behaviour. 2019;2(3):188-96.

39. Hildebrand M, Hansen BH, van Hees VT, Ekelund U. Evaluation of raw acceleration sedentary thresholds in children and adults. Scand J Med Sci Sport. 2017;27(12):1814-23.

40. Hildebrand M, Van Hees VT, Hansen BH, Ekelund ULF. Age Group Comparability of Raw Accelerometer Output from Wrist- and Hip-Worn Monitors. Med Sci Sports Exerc. 2014;46(9):181624.

41. Gu F, Khoshelham K, Shang J, Yu F, Wei Z. Robust and Accurate Smartphone-Based Step Counting for Indoor Localization. IEEE Sens J. 2017;17(11):3453-60.

42. Collings PJ, Brage S, Ridgway CL, Harvey NC, Godfrey KM, Inskip HM, et al. Physical activity intensity, sedentary time, and body composition in preschoolers. Am J Clin Nutr. 2013;97(5):1020-8.

43. Gomersall SR, Rowlands AV, English C, Maher C, Olds TS. The activitystat hypothesis. Sports medicine. 2013;43(2):135-49.

44. Ortega FB, Cadenas-Sánchez C, Sánchez-Delgado G, Mora-González J, Martínez-Téllez B, Artero EG, et al. Systematic review and proposal of a field-based physical fitness-test battery in preschool children: the PREFIT battery. Sports medicine. 2015;45(4):533-55.

45. Gustafsson BM, Gustafsson PA, Proczkowska-Bjorklund M. The Strengths and Difficulties Questionnaire (SDQ) for preschool children-a Swedish validation. Nord J Psychiatry. 2016;70(8):567-74.

46. van Hees VT, Sabia S, Jones SE, Wood AR, Anderson KN, Kivimaki M, et al. Estimating sleep parameters using an accelerometer without sleep diary. Scientific reports. 2018;8(1):12975.

47. Salomonsson B, Sleed M. The Ages \& Stages Questionnaire: Social-Emotional: A validation study of a mother-report questionnaire on a clinical mother-infant sample. Infant Ment Health J. 2010;31(4):412-31.

48. Cole TJ, Bellizzi MC, Flegal KM, Dietz WH. Establishing a standard definition for child overweight and obesity worldwide: international survey. BMJ. 2000;320(7244):1240-3.

49. Cadenas-Sanchez C, Martinez-Tellez B, Sanchez-Delgado G, Mora-Gonzalez J, Castro-Piñero J, Löf M, et al. Assessing physical fitness in preschool children: Feasibility, reliability and practical recommendations for the PREFIT battery. Journal of Science Medicine in Sport. 2016;19(11):910-5.

50. Ward D, Mazzucca S, McWilliams C, Hales D. Use of the Environment and Policy Evaluation and Observation as a Self-Report Instrument (EPAO-SR) to measure nutrition and physical activity 
environments in child care settings: validity and reliability evidence. Int J Behav Nutr Phy. 2015;12(1):124.

51. Finch TL, Girling M, May CR, Mair FS, Murray E, Treweek S, et al. Improving the normalization of complex interventions: part 2 - validation of the NoMAD instrument for assessing implementation work based on normalization process theory (NPT). BMC Med Res Methodol. 2018;18(1):135.

52. Finch T, Girling M, May C, Mair F, Murray E, Treweek S, et al. NoMAD: implementation measure based on Normalization Process Theory.[Measurement instrument]. 2015.

\section{Figures}

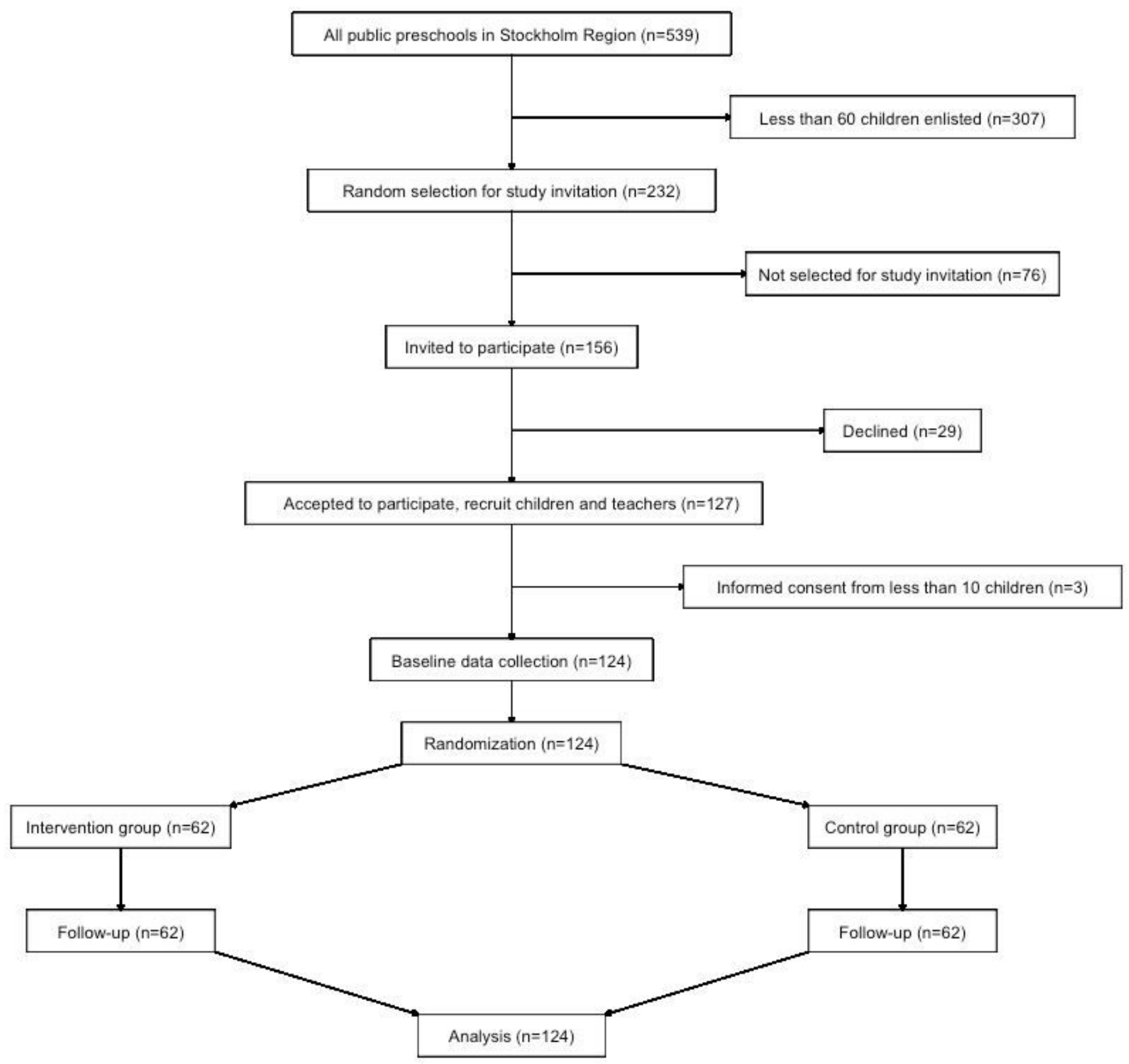

Figure 1 
Flow-chart of study recruitment, randomization and follow-up

\begin{tabular}{|c|c|c|c|c|c|c|}
\hline \multirow[b]{3}{*}{ TIMEPOINT ${ }^{* *}$} & \multicolumn{6}{|c|}{ STUDY PERIOD } \\
\hline & \multirow{2}{*}{$\begin{array}{c}\text { Enrolment } \\
0\end{array}$} & \multirow{2}{*}{$\frac{\text { Allocation }}{0}$} & \multicolumn{3}{|c|}{ Post-allocation } & \multirow{2}{*}{$\begin{array}{c}\text { Close-out } \\
\begin{array}{c}\text { Post- } \\
\text { intervention }\end{array} \\
\end{array}$} \\
\hline & & & 0 & 3 month & 6 month & \\
\hline \multicolumn{7}{|l|}{ ENROLMENT: } \\
\hline \multirow{2}{*}{$\begin{array}{l}\text { Informed consent } \\
\text { Eligibility screen }\end{array}$} & $\mathrm{X}$ & & & & & \\
\hline & $\mathrm{X}$ & & & & & \\
\hline Allocation & & $x$ & & & & \\
\hline \multicolumn{7}{|l|}{ INTERVENTIONS: } \\
\hline \multicolumn{7}{|l|}{ [Intervention] } \\
\hline \multicolumn{7}{|l|}{ [Control] } \\
\hline \multicolumn{7}{|l|}{ ASSESSMENTS: } \\
\hline \multirow{2}{*}{$\begin{array}{r}\text { Accelerometer } \\
\text { Height, weight and } \\
\text { waist circumference }\end{array}$} & $\mathrm{x}$ & & & & $\mathrm{X}$ & \\
\hline & $\mathrm{X}$ & & & & $\mathrm{X}$ & \\
\hline \multirow{5}{*}{$\begin{array}{r}\text { Handgrip strength } \\
\text { Parental demography } \\
\text { questionnaire } \\
\text { Parental } \\
\text { measurement week } \\
\text { questionnaire } \\
\text { Strength and } \\
\text { difficulty and sleep } \\
\text { questionnaire } \\
\text { Intervention weekly } \\
\text { follow up } \\
\text { questionnaire }\end{array}$} & $\mathrm{X}$ & & & & $\mathrm{X}$ & \\
\hline & $\mathrm{X}$ & & & & & \\
\hline & $\mathrm{X}$ & & & & $\mathrm{X}$ & \\
\hline & $\mathrm{X}$ & & & & $\mathrm{X}$ & \\
\hline & & & & & & \\
\hline $\begin{array}{r}\text { Active transport } \\
\text { questionnaire }\end{array}$ & & & & & & $\mathrm{X}$ \\
\hline $\begin{array}{r}\text { Implementation } \\
\text { questionnaire }\end{array}$ & & & & & & $\mathrm{X}$ \\
\hline $\begin{array}{r}\text { policy Evaluation and } \\
\text { Observation }\end{array}$ & $\mathrm{X}$ & & & $\mathrm{X}$ & $\mathrm{x}$ & \\
\hline
\end{tabular}

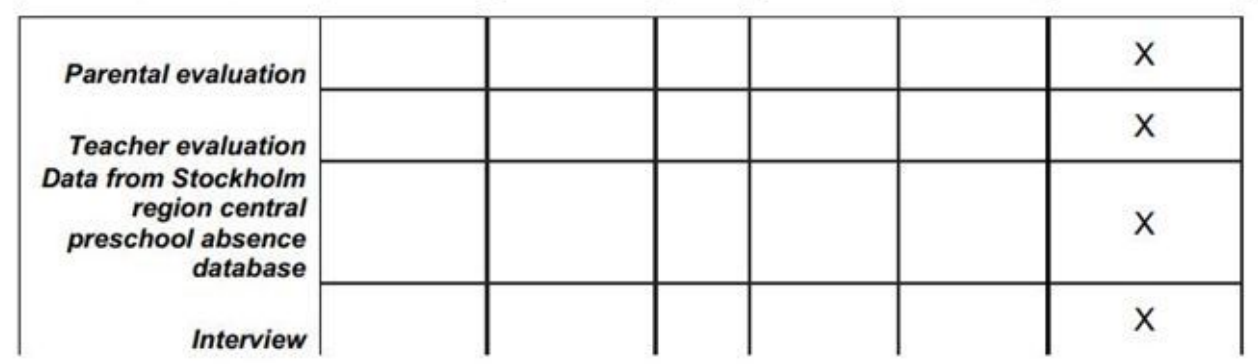

Figure 2

SPIRIT figure for schedule of enrollment, intervention and assessment 
This is a list of supplementary files associated with this preprint. Click to download.

- Additionalfile1.docx

- Additionalfile2.pdf

- Additionalfile3.docx

- Additionalfile4.do

- Additionalfile5.r

- Additionalfile6.xlsx

- Additionalfile7.docx

- Additionalfile8.pdf

- Additionalfile9.pdf

- Additionalfile10.pdf

- Additionalfile11.pdf

- Additionalfile12.pdf

- Additionalfile13.pdf

- Additionalfile14.pdf

- Additionalfile15.pdf 